\title{
Enhancement of Available Transfer Capability with Facts Device in the Competitive Power Market
}

\author{
Bairavan Veerayan Manikandan', Sathiasamuel Charles Raja ${ }^{2}$, Paramasivam Venkatesh ${ }^{2}$ \\ ${ }^{1}$ Mepco Schlenk Engineering College, Sivakasi, India \\ ${ }^{2}$ Thiagarajar College of Engineering, Madurai, India \\ E-mail:bvmani73@yahoo.com,\{charlesrajas,pveee\}@tce.edu \\ Received December 21, 2009; revised February 9, 2010; accepted February 12, 2010
}

\begin{abstract}
In order to facilitate the electricity market operation and trade in the restructured environment, ample transmission capability should be provided to satisfy the demand of increasing power transactions. The conflict of this requirement and the restrictions on the transmission expansion in the restructured electrical market has motivated the development of methodologies to enhance the Available Transfer Capability (ATC) of the existing transmission grids. The insertion of FACTS devices in electrical systems seems to be a promising strategy to enhance ATC. In this paper, the viability and technical merits of boosting ATC using Thyristor Controlled Series Compensator (TCSC) is being analyzed. The work has been carried out on IEEE 30 bus and IEEE 118 bus systems. Bilateral and multilateral transactions are considered. Particle Swarm Optimization (PSO) algorithm and Genetic Algorithm (GA) are employed to obtain the optimal settings of TCSC.
\end{abstract}

Keywords: Available Transfer Capability, Flexible AC Transmission Systems, Particle Swarm Optimization, Genetic Algorithm, Power Transfer Distribution Factors, Thyristor Controlled Series Compensator

\section{Introduction}

Deregulation of the electric industry throughout the world aims at creating competitive markets to trade electricity, which generates a host of new technical challenges to market participants and power system researchers. For transmission networks, one of the major consequences of the non-discriminatory open-access requirement is a substantial increase of power transfers, which demand adequate available transfer capability [1] to ensure all economic transactions. Researchers have proposed various methods to evaluate ATC [2-4]. Sufficient ATC should be guaranteed to support free market trading and maintain an economical and secure operation over a wide range of system conditions. However, tight restrictions on the construction of new facilities due to the increasingly difficult economic, environmental, and social problems, have led to a much more intensive shared use of the existing transmission facilities by utilities and independent power producers (IPPs). These concerns have motivated the development of strategies and methodologies to boost the ATC of the existing transmission networks.

Aimed at this problem, various ATC enhancement approaches have been proposed, where adjusting terminal voltage of generators and taps changing of onload tap changer (OLTC), particularly rescheduling generator outputs, are considered as major control measures for ATC boosting. On the other hand, it is highly recognized that, with the capability of flexible power-flow control and rapid action, flexible ac transmission systems (FACTS) technology has a wide spectrum of impacts on the way the transmission system operates, in particular with respect to thermal, voltage, and stability constraints [5].

ATC values are always limited ultimately by heavily loaded circuits and/or nodes with relatively low voltage, with the increase of system loading. FACTS concept makes it possible to use circuit reactance, voltage magnitude, and phase angle as controls to redistribute line flow and regulate nodal voltage, thereby mitigating the critical situation. In addition, partly due to the physical constraints on circuit impedance and phase angle of nodal voltage, most high-voltage transmission lines are operating far below their thermal rating [6]. By the control of line reactance and voltage phase angle, FACTS technology enables line loading to increase flexibly, in some cases, all the way up to thermal limits. Therefore, theoretically it can offer an effective and promising alternative to conventional methods for ATC enhancement. 
Undoubtedly, it is very important and imperative to carry out studies on exploitation of FACTS technology to enhance the ATC [7].

In this paper, ATC is calculated using ACPTDF in Combined Economic Emission Dispatch (CEED) environment $[8,9]$ and an attempt is made to enhance Available Transfer Capability using TCSC i.e. Thyristor controlled series compensator [10,11]. TCSC is connected in series with the line conductors to compensate for the inductive reactance of the line. Both bilateral and multilateral transactions are considered. Population based, cooperative and competitive stochastic search algorithms are very popular in the recent years in the research arena of computational intelligence. Some well established search algorithms such as PSO [12,13] and GA [14,15] are successfully implemented to solve simple and complex problems efficiently and effectively. Population based search approach in GA is motivated by evolution as seen in nature. PSO, on the other hand, is motivated from the simulation of social behavior. The optimal settings of TCSC are obtained using PSO and GA. The results are illustrated on both IEEE 30 bus and IEEE 118 bus systems.

\section{Available Transfer Capability}

Available Transfer Capability (ATC) is a measure of the transfer capability remaining in the physical transmission network for further commercial activity over and above the already committed uses [1]. ATC can be expressed as:

$$
\text { ATC }=\text { TTC }- \text { Existing Transmission Commitments }
$$

where, Total Transfer Capability (TTC) is defined as the amount of electric power that can be transferred over the interconnected transmission network or particular path or interface in a reliable manner while meeting all of a specific set of defined pre and post contingency conditions.

ATC at base case, between bus $m$ and bus $n$ using line flow limit (thermal limit) criterion is mathematically formulated using ACPTDF (or) PTDF as

$$
A T C_{m n}=\min \left\{T_{i j, m n}\right\}, i j \in N_{L}
$$

where $T_{i j, m n}$ denotes the transfer limit values for each line in the system. It is given by

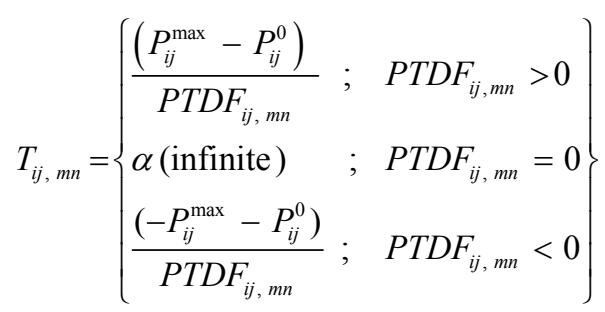

where

$$
P_{i j}^{\max } \text { is the MW power limit of a line between bus } i
$$

and $j$

$P_{i j}^{\mathrm{o}}$ is the base case power flow in line between bus $i$ and $j$.

$P T D F_{i j, m n}$ is the power transfer distribution factor for the line between bus $i$ and $j$ when a transaction is taking place between bus $m$ and $n$.

$N_{L}$ is the total number of lines.

The optimal settings of generators under CEED environment are considered as a base case power flow.

\subsection{CEED Problem Formulation}

Optimization of CEED problem has been mathematically formulated and is given by the following equation:

$$
\phi=\min \sum_{i=1}^{N g} f_{i}(F C, E C)
$$

where $\varphi$ is the optimal cost of generation (US\$/h) and $N_{g}$ represents the number of generators connected in the network.

The cost is optimized within the following constraints: The power system constraint is given as follows

$$
\sum_{i=1}^{N g} P_{g i}=P_{d}+P_{l}
$$

where $P_{d}$ is the total load of the system and $P_{l}$ is the transmission losses of the system.

The power flow equation of the power network

$$
g(|v|, \theta)=0
$$

The inequality constraint on real power generation of each generator $i$

$$
P_{g i}^{\min } \leq P_{g i} \leq P_{g i}^{\max }
$$

where $P_{g i}^{\min }$ and $P_{g i}^{\max }$ are minimum and maximum value of real power allowed at generator $i$ respectively. The inequality constraint on voltage of each PQ bus

$$
V_{i}^{\min } \leq V_{i} \leq V_{i}^{\max }
$$

where $V_{i}^{\min }$ and $V_{i}^{\max }$ are minimum and maximum voltage at bus $i$ respectively.

Power limit on transmission line

$$
M V A f_{p q} \leq M V A f_{p q}^{\max }
$$

where $M V A f_{p q}^{\max }$ is the maximum rating of transmission line connected between buses $p$ and $q$.

Total fuel cost of generation FC (US\$/h) in terms of control variables of generator powers can expressed as,

$$
F C=\sum_{i=1}^{N g}\left(a_{i} P_{g i}^{2}+b_{i} P_{g i}+c_{i}\right)
$$

where $P_{g i}$ is the real power output of an $i^{\text {th }}$ generator and $a_{i}, b_{i}, c_{i}$ are the fuel cost curve coefficients. 
Total emission of generation EC $(\mathrm{lb} / \mathrm{h})$ can be expressed as,

$$
E C=\sum_{i=1}^{N g}\left(\alpha_{i} P_{g i}^{2}+\beta_{i} P_{g i}+\gamma_{i}\right)
$$

where $\alpha_{i}, \beta_{i}$ and $\gamma_{i}$ are emission coefficients.

The bi-objective optimization problem (4) is converted into single optimization problem by introducing price penalty factor, $h$, which blends fuel cost with emission,

$$
\text { Minimize } \phi=(F C+h E C)
$$

CEED optimization problem is solved using evolutionary programming and more information is also available in the papers [8,9]. In most of the developing countries, the restructuring process of power industry is in the infant stage. Still the structure is vertically integrated but they purchase power from Independent Power Producers (IPP) to meet the growing demand. Hence, the regional transmission operator is responsible for the redispatch of generator power by considering the physical limits of the system and emissions standards. Also, there are some power markets which support both bilateral transactions based on ATC and centralized dispatch based on bids. In these markets, assured firm transactions are implemented first and then they will follow centralized dispatch mechanism with the remaining transfer capacity. Therefore the proposed CEED based ATC calculation and enhancement method can be well suited for such cases described above.

\subsection{ACPTDF Formulation}

The AC power transfer distribution factors proposed for calculation of ATC [4] were used to find various transmission system quantities for a change in MW transaction at different operating conditions.

Consider a bilateral transaction $t_{k}$ between a seller bus $m$ and buyer bus $n$. Line 1 carries the part of the transacted power and is connected between buses $i$ and $j$. For a change in real power, transaction among the above buyer and seller by $\Delta t_{k} \mathrm{MW}$, if the change in a transmission line quantity $q_{1}$ is $\Delta q_{1}$, power transfer distribution factors can be defined as,

$$
P T D F_{i j, m n}=\frac{\Delta q_{l}}{\Delta t_{k}}
$$

The transmission quantity $q_{l}$ can be either real power flow from bus $i$ to $j\left(P_{i j}\right)$ (or) real power flow from bus $j$ to bus $i\left(P_{j i}\right)$. The above factors have been proposed to compute at a base case load flow with results using sensitivity properties of NRLF Jacobian. Consider full Jacobian in polar coordinates $\left[J_{T}\right]$, defined to include all the buses except slack (including $\Delta \mathrm{Q}-\Delta \mathrm{V}$ equations also for PV buses).

$$
\left[\begin{array}{l}
\Delta \delta \\
\Delta V
\end{array}\right]=\left[\begin{array}{ll}
\frac{\partial P}{\partial \delta} & \frac{\partial P}{\partial V} \\
\frac{\partial Q}{\partial \delta} & \frac{\partial Q}{\partial V}
\end{array}\right]^{-1}\left[\begin{array}{c}
\Delta P \\
\Delta Q
\end{array}\right]=\left[J_{T}\right]^{-1}\left[\begin{array}{c}
\Delta P \\
\Delta Q
\end{array}\right]
$$

In a base case load flow, if only one of the $k^{\text {th }}$ bilateral transactions is changed by $\Delta t_{k} \mathrm{MW}$, only the following two entries in the mismatch vector on RHS of (14) will be non zero.

$$
\Delta P_{i}=\Delta t_{k} \quad \Delta P_{j}=-\Delta t_{k}
$$

With the above mismatch vector elements, the change in voltage angle and magnitude at all buses can be computed from (14) \& (15) and, hence, the new voltage profile can be calculated. These can be utilized to compute all the transmission quantities $q_{1}$ and hence the corresponding changes in these quantities $\Delta q_{1}$ from the base case. Once the $\Delta q_{1}$ for all the lines corresponding to a change in transaction $\Delta t_{k}$ is known, PTDFs can be obtained from (13).

\section{Overview of PSO and GA}

\subsection{Particle Swarm Optimization}

The PSO is a population-based optimization method first proposed by Kennedy and Eberhart [12]. PSO technique finds the optimal solution using a population of particles. PSO is developed through simulation of bird flocking in two-dimensional space. The position of each agent is represented in $X-Y$ plane with position $\left(S_{x}, S_{y}\right), V_{x}$ (velocity along $X$-axis), and $V_{y}$ (velocity along $Y$-axis). Modification of the agent position is realized by the position and velocity information. Bird flocking optimizes a certain objective function. Each agent knows its best value so far, called ' $P_{\text {best }}$, , which contains the information on position and velocities. This information is the analogy of personal experience of each agent. Moreover, each agent knows the best value so far in the group, ' $G_{\text {best }}$ ' among all ' $P_{\text {best }}$ '. This information is the analogy of knowledge, how the other neighbouring agents have performed. Each agent tries to modify its position by considering current positions $\left(S_{x}, S_{y}\right)$, current velocities $\left(V_{x}, V_{Y}\right)$, the individual intelligence $\left(P_{\text {best }}\right)$, and the group intelligence $\left(G_{\text {best }}\right)$.

The following equations are utilized, in computing the position and velocities, in the $X$ - $Y$ plane:

$$
\begin{gathered}
V_{i}^{k+1}=W \times V_{i}^{k}+\left[C_{1} \times \text { rand }_{1} \times\left(P_{\text {best }_{i}}-S_{i}^{k}\right)\right] \\
+\left[C_{2} \times \operatorname{rand}_{2} \times\left(G_{\text {best }}-S_{i}^{k}\right)\right] \\
S_{i}^{k+1}=S_{i}^{k}+V_{i}^{k+1}
\end{gathered}
$$

where, $V_{i}^{k+1}$ is the velocity of $i^{t h}$ individual at $(K+1)^{t h}$ 
iteration, $V_{i}^{k}$ is the velocity of $i^{\text {th }}$ individual at $k^{\text {th }}$ iteration, $W$ is the inertia weight, $C_{l}, C_{2}$ are the positive constants having values $(0,2.5)$, rand $_{1}$, rand $_{2}$ are the random numbers selected between 0 and $1, P_{\text {best }}$ is the best position of the $i^{\text {th }}$ individual, $G_{\text {best }}$ is the best position among the individuals (group best) and $S_{i}^{k}$ is the position of $i^{\text {th }}$ individual at $k^{\text {th }}$ iteration. The acceleration coefficients $C_{1}$, and $C_{2}$ control how far a particle will move in a single iteration. Typically, these are both set to a value of 2.5 .

The velocity of each particle is modified according to (16) and the minimum and maximum velocity of each variable in each particle is set within the limits of $V_{\min }$ and $V_{\max }$ respectively. The position is modified according to (17). The inertia weight factor ' $W$ ' is modified using (18) to enable quick convergence.

$$
W=W_{\max }-\frac{\left(W_{\max }-W_{\min }\right)}{\text { iter }_{\max }} \times \text { iter }
$$

where $W_{\max }$ is the initial value of inertia weight equal to $0.9, W_{\min }$ is the final value of inertia weight equal to 0.4 , iter is the current iteration number and iter $_{\max }$ is the maximum iteration number. Small values of $w$ result in more rapid convergence usually on a suboptimal position, while a too large value may prevent divergence.

The PSO system combines two models: A social-only model and the cognition-only model. These models are represented by the velocity update, shown in (16). The second term in the velocity update equation $\left[C_{1} \times\right.$ rand $\left._{1} \times\left(P_{\text {best }_{i}}-S_{i}^{k}\right)\right]$ is associated with cognition since it only takes into account the particle's own experiences. The third term in the velocity update equation $\left[C_{2} \times\right.$ rand $\left._{2} \times\left(G_{\text {best }}-S_{i}^{k}\right)\right]$ represents the social interaction between the particles. It suggests that individuals ignore their own experience and adjust their behavior according to the successful beliefs of individual in the neighborhood.

\subsection{Genetic Algorithm}

GAs has been extensively used in power system optimization problems. GAs are search algorithms based on the mechanics of natural selection and natural genetics. GAs are different from other optimization methods. GAs search from a population of points, not from a single point. GAs can therefore discover a globally optimal point. GAs can deal with non-smooth, non-continuous and non-differentiable functions that are the real-life optimization problems. GAs use probabilistic transition rules to select generations, not deterministic rules, so they can search a complicated and uncertain area to find the global optimum. However, to make GAs more practicable, the problems of memory and computing time arising from the coding of large number of variables in real life systems need to be solved. The steps involved in simple GA are Initial population generation, Fitness evaluation, Selection, Crossover and Mutation.

In this work, Real Coded Genetic Algorithm (RCGA) with specialized crossover operator called Simulated Binary crossover (SBX) and polynomial mutation is employed. Simulated Binary Crossover creates children solutions in proportion to the difference in parent solutions. The following steps are followed to create two children solutions from two parents:

- Choose a random number $u_{i}, \in[0,1]$

- Calculate $\beta_{q i}$ as given in the below equation

$$
\beta_{q i}= \begin{cases}\left(2 u_{i}\right)^{\frac{1}{\eta_{c}+1}}, & u_{i} \leq 0.5 \\ \left(\frac{1}{2\left(1-u_{i}\right)}\right)^{\frac{1}{\eta_{c}+1}}, & \text { otherwise }\end{cases}
$$

where, $\beta_{q i}$ is the spread factor and is defined as the ratio of the absolute difference in offspring values to that of the parents. $\eta_{c}$ is the crossover index.

Then compute the offspring $x_{i}^{(1, t+1)} \& x_{i}^{(2, t+1)}$ as,

$$
\begin{aligned}
& x_{i}^{(1, t+1)}=0.5\left[\left(1+\beta_{q i}\right) x_{i}^{(1, t)}+\left(1-\beta_{q i}\right) x_{i}^{(2, t)}\right] \\
& x_{i}^{(2, t+1)}=0.5\left[\left(1-\beta_{q i}\right) x_{i}^{(1, t)}+\left(1+\beta_{q i}\right) x_{i}^{(2, t)}\right]
\end{aligned}
$$

Newly generated offspring undergo polynomial mutation operation. Like in the SBX operator, the probability distribution can also be a polynomial function, instead of a normal distribution. The new offspring $y_{i}^{(1, t+1)}$ is determined as follows,

$$
y_{i}^{(1, t+1)}=x_{i}^{(1, t+1)}+\left(x_{i}^{U}-x_{i}^{L}\right) \overline{\delta_{i}}
$$

where $x_{i}^{U}$ and $x_{i}^{L}$ are the upper and lower limit values. The parameter $\overline{\delta_{i}}$ is calculated from the polynomial probability distribution.

$$
\begin{aligned}
& P(\delta)=0.5\left(\eta_{m}+1\right)(1-|\delta|)^{\eta_{m}} \\
& \text { and } \\
& \overline{\delta_{i}}= \begin{cases}\left(2 r_{i}\right)^{1 /\left(\eta_{m}+1\right)}-1, & \text { if } r_{i}<0.5 \\
1-\left[2\left(1-r_{i}\right)\right]^{1 /\left(\eta_{m}+1\right)}, & \text { if } r_{i} \geq 0.5\end{cases}
\end{aligned}
$$

where $\eta_{m}$ is the mutation index. In this operator the shape of the probability distribution is directly controlled by the external parameter $\eta_{m}$ and distribution is not dynamically changed with generations. Newly generated individuals replace their parents and forms the parents for the next generation. The iterative procedure can be terminated when any one of the following criteria is met i.e., an acceptable solution has been reached, a state with no further improvement in solution is reached, control 
variables has converged to a stable state or a pre-defined number of iterations have been completed.

\section{Thyristor Controlled Series Compensator}

A TCSC is a series-controlled capacitive reactance that can provide continuous control of power on the AC line over a wide range. It can be operated in both capacitive and inductive modes. In capacitive mode, it reduces the transfer reactance between the buses at which the line is connected, thus increasing the maximum power that can be transmitted and reducing the effective active and reactive power losses. From the system viewpoint, the principle of variable-series compensation is simply to increase the fundamental-frequency voltage across a fixed capacitor (FC) in a series-compensated line through appropriate variation of the firing angle, $\alpha$. This enhanced voltage changes the effective value of the series- capacitive reactance

The basic conceptual TCSC module comprises a series capacitor, $\mathrm{C}$, in parallel with a thyristor-controlled reactor, $\mathrm{L}_{\mathrm{s}}$ as shown in Figure 1. However, a practical TCSC module also includes protective equipment normally installed with series capacitors.

The model of a transmission line with a TCSC connec ted between bus- $\mathrm{i}$ and bus-j is shown in Figure 2 .

In this work, TCSC is modelled as a variable reactance whose value varies from $-0.8 X_{L}$ to $+0.2 X_{L}$ in order to avoid over compensation of the line. $\mathrm{X}_{\mathrm{L}}$ is the reactance of the line in which TCSC is connected.

\section{Problem Formulation}

The aim of the optimization is to perform the best

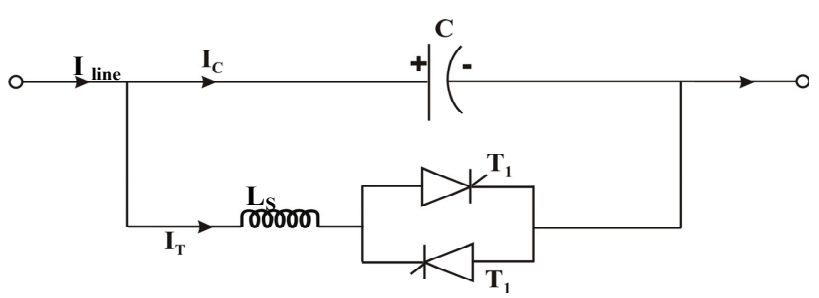

Figure 1. TCSC basic module.

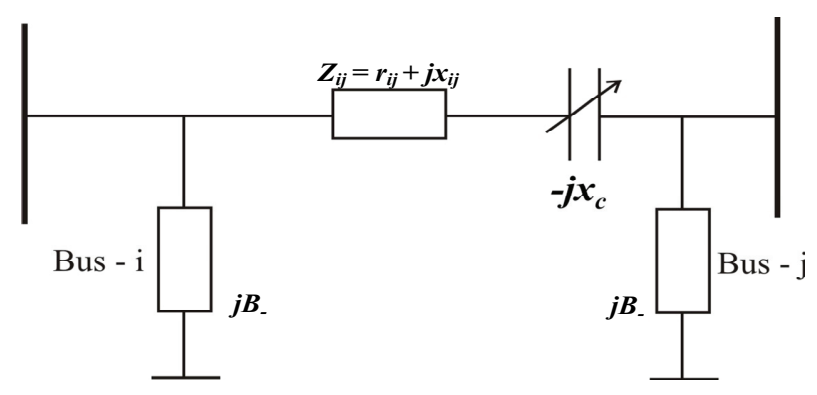

Figure 2. Model of TCSC. utilization of the existing transmission lines. The objective is to maximize the ATC i.e., uncommitted active transfer capacity of the prescribed interface, when a transaction is taking place between a seller bus (m) and buyer bus (n). It is represented as

$$
\text { Maximize } A T C_{m n} p
$$

where $A T C_{m n}$ is given by Equation (2). The optimization problem is solved subject to the following FACTS device constraint (24) and power flow constraints (5-9).

$$
-0.8 X_{L} \leq X_{T C S C} \leq+0.2 X_{L} p
$$

\section{Algorithm}

The basic steps involved in enhancing ATC values with TCSC device using PSO and GA algorithm are given below:

Step 1: Read the system input data.

Step 2: Run a base case load flow in CEED environment and determine the optimal settings of generators $[8,9]$.

Step 3: Consider wheeling transactions (tk).

Step 4: Compute AC power transfer distribution factors as per (13).

Step 5: Take transactions as variables, line flow, real and reactive power limits of generators as constraints and compute the feasible wheeling transactions determine the ATC as per (2).

Step 6: Find the limiting element in the system buses i.e., that carry power close to thermal limit

Step 7: Place TCSC in the limiting element.

Step 8: Run PSO algorithm and GA separately to obtain the settings of TCSC.

Step 9: Calculate ATC after incorporating TCSC

Step 10: Is any other transaction has to be carried out, then consider the next transaction and go to step 3, otherwise stop the procedure.

\section{Results and Discussion}

The simulation studies are carried out on Intel Pentium Dual Core, 2.40 GHZ system in MATLAB 7.3 environment.

The work has been carried out on IEEE 30 bus and IEEE 118 bus systems. For the ATC determination, generators setting are obtained from CEED environment as explained by the authors in $[8,9]$. Thermal limit of each line is considered as a constraint and reactive power demand at load buses has been taken as constant. For running the PSO algorithm, the initial population of individuals are created by satisfying the constraints. For each individual in the population, the fitness function, velocity 
Table.1. ATC in MW - with and without TCSC using PSO.

\begin{tabular}{cccccccc}
\hline System & Method & $\begin{array}{c}\text { Transaction } \\
\text { between buses }\end{array}$ & $\begin{array}{c}\text { ATC } \\
\text { Without } \\
\text { TCSC } \\
\text { (MW) }\end{array}$ & $\begin{array}{c}\text { ATC With } \\
\text { TCSC } \\
\text { (MW) }\end{array}$ & $\begin{array}{c}\text { Settings of } \\
\text { TCSC } \\
\text { (p.u.) }\end{array}$ & $\begin{array}{c}\text { Position of } \\
\text { TCSC }\end{array}$ & $\begin{array}{c}\text { Execution time } \\
\text { (sec) }\end{array}$ \\
\hline \multirow{2}{*}{$\begin{array}{c}\text { IEEE 30 } \\
\text { Bus }\end{array}$} & ACPTDF & $2-28$ & 22.970 & 26.553 & 0.0300 & $6-28$ & 103.056707 \\
& & $5-23$ & 23.901 & 23.935 & 0.1350 & $23-24$ & 102.823764 \\
\hline
\end{tabular}

Table 2. ATC in MW - with and without TCSC using GA.

\begin{tabular}{cccccccc}
\hline System & Method & $\begin{array}{c}\text { Transaction } \\
\text { between buses }\end{array}$ & $\begin{array}{c}\text { ATC With- } \\
\text { out TCSC } \\
\text { (MW) }\end{array}$ & $\begin{array}{c}\text { ATC With } \\
\text { TCSC } \\
\text { (MW) }\end{array}$ & $\begin{array}{c}\text { Settings of } \\
\text { TCSC } \\
\text { (p.u.) }\end{array}$ & $\begin{array}{c}\text { Position of } \\
\text { TCSC }\end{array}$ & $\begin{array}{c}\text { Execution time } \\
\text { (sec) }\end{array}$ \\
\hline \multirow{2}{*}{$\begin{array}{c}\text { IEEE 30 } \\
\text { Bus }\end{array}$} & ACPTDF & $2-28$ & 22.970 & 26.1473 & 0.0277 & $6-28$ \\
& & $5-23$ & 23.901 & 23.8743 & 0.1332 & $23-24$ \\
\hline
\end{tabular}

updation and new population creation are done as explained in Subsection 3.1.1.

The network and line datas for IEEE 30 bus system IEEE 118 bus system is taken from [16]. There are 6 generators and 41 lines in the IEEE 30 bus system. In IEEE 118 bus system, there are 13 generators and 99 lines. For each considered transaction, TCSC is placed in the most limiting line i.e., line flow close to thermal limit. Algorithms are run for 100 iterations.

Three bilateral transactions and one multilateral transaction are considered for IEEE 30 bus system. Two bilateral and one multilateral transaction are considered for IEEE 118 bus system.

\subsection{Bilateral Transaction}

The position of TCSC, its settings from PSO algorithm and the ATC values before and after incorporating TCSC are shown in Table 1. The steps involved in simple GA i.e., initial population generation, fitness evaluation, selection, crossover and mutation are carried out for the considered transactions and the results are given in Table 2.

For the IEEE 118 bus system, two bilateral transactions between buses i.e., (49-100) and (1-118) are considered. The results obtained with TCSC using PSO and GA are shown in Figure 3.

For the PSO algorithm, the settings of TCSC are 0.0185 p.u for the transaction (49-100) and 0.0240 p.u for transaction (1-118). TCSC is placed in series with the limiting line 81-80 for transaction (49-100) and for the transaction (1-118), it is placed in the limiting line 75-118. The execution time is 139.047090 seconds for transaction (49-100) and 137.654722 for transaction (1-118).
With GA, the TCSC settings, placement and execution time for the transaction (49-100) are 0.0148 p.u, line 81-80 and 146.347801 seconds respectively. Similarly, for the transaction (1-118), the values are 0.0229 p.u, line 75-118 and 145.778420 seconds respectively.

\subsection{Multilateral Transaction}

The results for multilateral transaction using PSO are shown in Figure 4. For the multilateral transaction between buses i.e., $(2,11)-(28,26)$ considered in IEEE30 bus system, TCSC is placed in the line between buses 25-27 and its setting is 0.1044 p.u. The time taken for completing the execution is 102.810250 seconds. For the multilateral transaction between buses i.e., $(25,59,46)-$ $(89,100,103,111)$ considered in IEEE 118 bus system, TCSC is placed in the line between buses 100-103 and its setting is 0.0262 p.u. The execution time is 136 . 225383 seconds.

The results for multilateral transaction using GA are shown in Figure 5. For the same multilateral transaction considered in IEEE30 bus system, TCSC is placed in the line between buses $25-27$ and its setting is 0.1040 p.u.

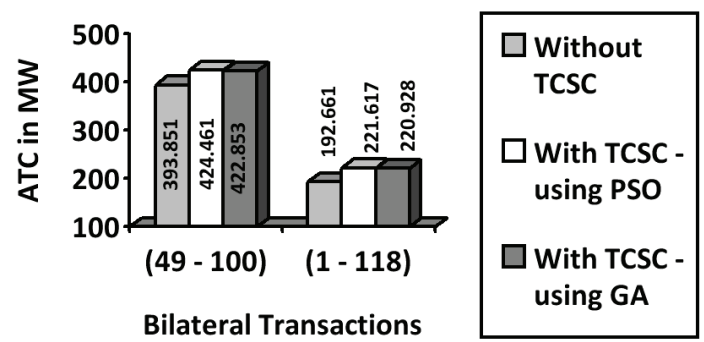

Figure 3. ATC results - using PSO and GA. 


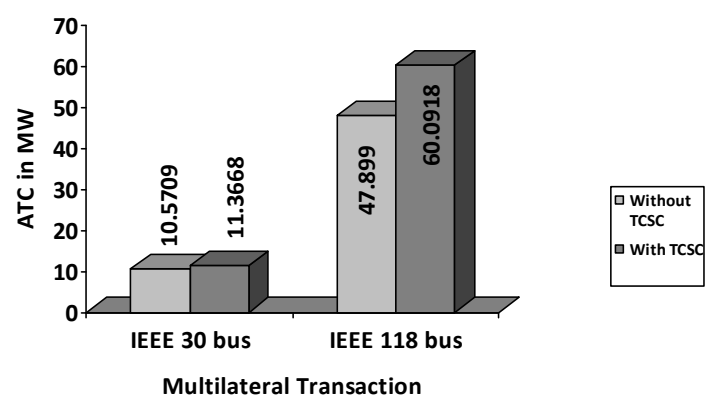

Figure 4. ATC for Multilateral transaction - PSO.

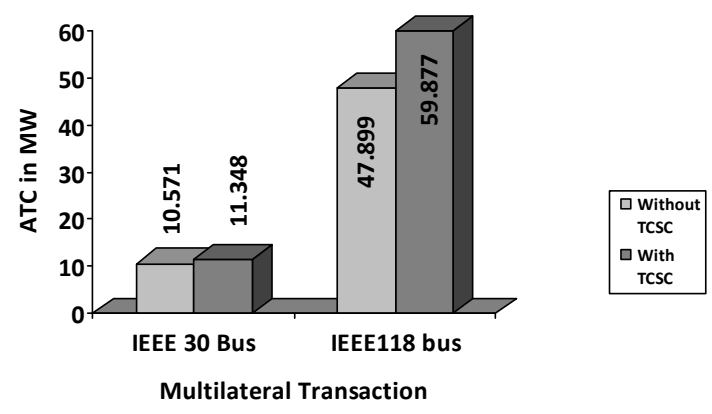

Figure 5. ATC for Multilateral transaction - GA.

The time taken for completing the execution is 103 . 120562 seconds. For the same multilateral transaction in IEEE 118 bus system, TCSC is placed in the line between buses $100-103$ and its setting is 0.0254 p.u. The execution time is 134.927363 seconds.

\section{Conclusions}

From the view point of operational planning, this paper evaluated the impact of FACTS device on ATC enhancement. The results demonstrated that the use of FACTS devices, particularly the TCSC can boost the ATC substantially. The considerable difference between ATC values with and without TCSC justifies that the FACTS technology can offer an effective and promising solution to boost the usable power transfer capability, thereby improving transmission services of the competitive electricity market. On using PSO and GA for the above problem, it is found that, PSO algorithm is providing very good enhanced result with minimum execution time compared to GA. But for the multilateral transaction, the GA results are very much closer to PSO results in all aspects including settings of TCSC and execution time. Both algorithms predicted the same limiting line for bilateral and multilateral transactions considered in IEEE test systems.

\section{References}

[1] "ATC Definitions and Determination, a Frame Work for Determining Available Transfer Capabilities of the In- terconnected Transmission Networks for a Commercially Viable Electricity Market," NERC Report, June 1996.

[2] J. Weber, "Efficient Available Transfer Capability Analysis Using Linear Method," PSERC Internet Seminar, Power World Corporation, Urbana, 2000.

[3] A. Kumar and S. C. Srivatsava, "AC Power Distribution Factors for allocation Power Transactions in a Deregulated Environment," IEEE Power Engineering Review, 2002, pp. 42-43.

[4] A. Kumar, S. C. Srivatsava and S. N. Singh, "ATC Determination in a Competitive Electricity Market Using AC Distribution Factors," Electrical Power components and Systems, Vol. 32, 2004, pp. 927-939.

[5] N. G. Hingorani and L. Gyugyi, "Understanding FACTS Concepts and Technology of Flexible AC Transmission Systems," 1st Indian Edition, IEEE Press, New York and Standard Publishers Distributors, Delhi, 2000.

[6] R. M. Mathur and R. K. Varma, "Thyristor Based FACTS Controllers for Electrical Transmission Systems," 1st Edition, John Wiley \& Sons Inc., 2002.

[7] Y. Xiao, Y. H. Song, C. C. Liu and Y. Z. Sun, "Available transfer capability enhancement using FACTS devices," IEEE Transactions on Power Systems, Vol. 18, No. 1, 2003, pp. 910-955.

[8] B. V. Manikandan, S. C. Raja, P. Venkatesh and P. S. Kannan, "Available Transfer Capability Determination in the Restructured Electricity Market," Electrical Power Components and Systems, Vol. 36, No. 9, 2008, pp. 941-959.

[9] P. Venkatesh, R. Gnanadass and N. P. Padhy, "Comparison and Application of Evolutionary Programming Techniques to Combined Economic Emission Dispatch With Line Flow Constraints," IEEE Transactions on Power Systems, Vol. 18, No. 2, 2003, pp. 688-697.

[10] A. Kazemi and B. Badrzadeh, "Modeling and Simulation of SVC and TCSC to Study Their Limits on Maximum Loadability Point," International Journal of Electrical Power and Energy Systems, Vol. 26, 2004, pp. 619-626.

[11] Y. Ou and C. Singh. "Improvement of Total Transfer Capability Using TCSC and SVC," IEEE Summer Power Meeting, Vancouver, Vol. 2, 2001, pp. 944-948.

[12] J. Kennady and R. Eberhart, "Particle Swarm Optimization," Proceedings of the IEEE International Conference on Neural Networks, Perth, 1995, pp. 1942-1948.

[13] M. A. Abido, "Optimal Power Flow using Particle Swarm Optimisation," International Journal of Electrical Power and Energy Systems, Vol. 24, 2002, pp. 563-571.

[14] S. Gerbex, R. Cherkaoui and A. J. Germond, "Optimal Location of Multi-Type FACTS Devices by Means of Genetic Algorithm," IEEE Transactions on Power Systems, Vol. 16, No. 3, 2001, pp. 537-544.

[15] S. Baskar, "Short Term Course on Evolutionary Multiobjective Optimization Algorithms (EMOA)," Procedings published by Department of Electrical and Electronics Engineering, Thiagarajar College of Engineering, Madurai, 2007.

[16] http://www.ee.washington.edu/research/pstca 